\title{
Estudio de fiabilidad, mantenibilidad y disponibilidad aplicado a grupos electrógenos prime
}

\author{
Reliability, maintainability and availability study applied to prime \\ generator sets.
}

César Marcelo Gallegos Londoño. ${ }^{1}$, Mayra Alexandra Viscaíno Cuzco. ${ }^{2}$ \& Sergio Raúl Villacrés Parra. ${ }^{3}$

Resumen.

DOI: https://doi.org/10.33262/concienciadigital.v3i3.1266

El presente estudio está enfocado en proporcionar una serie lógica de pasos necesarios para el cálculo de los indicadores de fiabilidad, mantenibilidad y disponibilidad, para el ejemplo se tomaron los registros de una base de datos de 51 generadores prime, se recolectaron los tiempos operativos entre fallos y los tiempos de reparación de dichos fallos por un período de 11 meses. La fiabilidad de un equipo depende de su diseño y construcción, para medir la fiabilidad es necesario registrar los tiempos operativos entre fallos, con estos tiempos calcular su tiempo medio, conocido por sus siglas en ingles MTBF (mean time between failure). El análisis de los tiempos de reparación de estos fallos y su media MTTR (mean time to repair) es la base de los estudios de la mantenibilidad. Las estrategias de mantenimiento deben enfocarse para extender los tiempos medios entre fallos y reducir los tiempos medios de reparación, con la finalidad de conservar la fiabilidad intrínseca de los equipos. Cabe mencionar que el mantenimiento preventivo no puede aumentar la fiabilidad por diseño de un dispositivo, si se realiza una buena planificación del mantenimiento podemos conservarla. Con la relación de estos indicadores mencionados se puede calcular la disponibilidad intrínseca. Otro aspecto importante es poder pronosticar la fiabilidad y la mantenibilidad, para ello es necesario tener un tratamiento estadístico con el estudio de funciones de distribución. El análisis de la fiabilidad, mantenibilidad y disponibilidad (análisis RAM por sus siglas en inglés) es una

\footnotetext{
${ }^{1}$ Escuela Superior Politécnica de Chimborazo, Facultad de Mecánica, Riobamba, Ecuador. cesar.gallegos@espoch.edu.ec

${ }^{2}$ Escuela Superior Politécnica de Chimborazo, Facultad de Mecánica, Riobamba, Ecuador. mayra.viscaino@espoch.edu.ec

${ }^{3}$ Escuela Superior Politécnica de Chimborazo, Facultad de Mecánica, Riobamba, Ecuador. sergio.villacres@espoch.edu.ec
} 
herramienta fundamental para predecir el rendimiento de equipos, provee información adecuada para anticiparnos a eventos de falla, esto proporcionará tiempo de reacción suficiente para tomar estrategias adecuadas. El pronóstico de la fiabilidad además es una herramienta muy útil cuando se requiere diseñar y construir equipos.

Palabras claves: Disponibilidad, Fiabilidad, Mantenibilidad, Análisis RAM, Mantenimiento.

\begin{abstract}
.
The present study is focused on providing a logical series of necessary steps in order to calculate the reliability, maintainability and availability indicators. For this example, records were taken from a database of 51 prime generators, operating times between failures and their respective repair time were collected within a period of 11 months. The reliability of an equipment depends on its design and construction, in order to measure the reliability, it is necessary to record the operating times between failures and use them to calculate the average time between failures, this times are used to calculate the meantime, known as MTBF (mean time between failure). The analysis of the failures repairing times and its mean MTTR (mean time to repair) is the basis of the maintainability studios. The maintenance strategies must focus in extending the mean time between failures and reduce the repairing mean times, with the purpose of keeping the equipment intrinsic reliability. It should be mentioned that preventive maintenance cannot increase a device reliability given by design, if a good preventive maintenance planning is carried out, the design reliability can be preserved. With these two indicators it is possible to determine the intrinsic availability of the equipment. Another important aspect is to be able to predict the reliability, availability and maintainability of the equipment, therefore it is necessary to have a statistical treatment with the study of distribution functions. Reliability, availability and maintainability analysis (RAM analysis) is a fundamental tool to predict equipment performance, it provides adequate information to anticipate failure events, this provides enough reaction time in order to take appropriate strategies. Reliability forecasting is a very useful tool when equipment design and build is required.
\end{abstract}

Keywords: Reliability, Availability, Maintainability, RAM Analysis, Maintenance.

\title{
Introducción.
}

Los grupos electrógenos Prime son máquinas que pueden generar energía eléctrica de forma continua ( 24 horas, 7 días), de respaldo o principales, logran trabajar al cien por ciento de su carga nominal (máxima potencia de diseño), con pausas únicamente para mantenimientos preventivos, trabajan además con carga variable, son utilizados en sitios 
donde la energía convencional no existe como campos petroleros aislados, sectores rurales, etc. El rendimiento de este tipo de equipos baja si los intervalos de las tareas de mantenimiento preventivo no se ejecutan con las frecuencias y los procedimientos adecuados, además de usar los materiales especificados por el fabricante, lo que ocasionaría un desgaste prematuro de sus componentes. El análisis de la fiabilidad mantenibilidad y disponibilidad conocido como análisis RAM por su sigla en inglés, Reliability, Availability, Maintainability, son importantes por varias razones como: el análisis de la seguridad de funcionamiento de los sistemas, el cumplimiento de sus funciones y el estudio de los costos de inversión, operación y mantenimiento durante el ciclo de vida del activo (Deparment Of Defense, 2005).

La fiabilidad es definida como la aptitud de un elemento para realizar una función requerida en condiciones indicadas y durante un período de tiempo especificado (CEN, 2018). La fiabilidad puede ser analizada en el campo estadístico como la probabilidad de que un equipo realice su función prevista en un período de tiempo establecido (NACHIAS, 1995). La fiabilidad es una característica de diseño, un equipo es tan fiable como fue diseñado y construido, las estrategias de mantenimiento deben enfocarse en conservar la fiabilidad intrínseca de los equipos (MoubraY, 2002).

La Mantenibilidad es también una característica de diseño, se expresa como la probabilidad que un elemento sea conservado o devuelto a un estado en que pueda cumplir su función, cuando estas actividades son realizadas de acuerdo con procedimientos y recursos establecidos (CEN, 2018) (Depatment Of Defense, 1966). Un equipo tiene mejor mantenibilidad cuando es más rápido y fácil realizar las tareas de mantenimiento, la mantenibilidad es un campo muy amplio, en este estudio se tomará en cuenta únicamente los tiempos empleados en tareas de mantenimiento correctivo. La disponibilidad es función de dos parámetros: de la frecuencia con la que se producen los fallos y de la velocidad con la que se los corrige. El tiempo de inactividad no programado de un equipo depende directamente de la rapidez para repararlo y restaurarlo y del tiempo utilizado para el soporte logístico necesario para realizar estas actividades. La definición normalizada de la disponibilidad se define como la capacidad de estar en un estado que pueda cumplir su función requerida en condiciones dadas y con los recursos necesarios (CEN, 2018) (Crespo, Moreu, \& Sánchez, 2004).Los factores que inciden en la disponibilidad se representan en la Figura 1.

Figura 1. Factores que afectan a la Disponibilidad

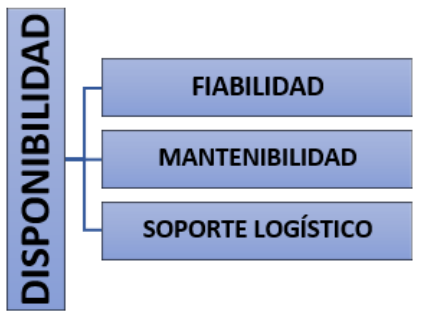

Fuente: Elaboración propia. 
Muchos factores afectan el desempeño de los equipos, entre otros: el contexto operacional donde trabajan los activos; el diseño y la calidad en su fabricación; cómo se los opera; cómo se los mantiene; la logística de mantenimiento, y los métodos de detección de fallos (BS-EN, 2016).

Para el cálculo de los indicadores de fiabilidad, mantenibilidad y disponibilidad, como se mencionó anteriormente es necesario recolectar los tiempos operativos entre fallos y los tiempos de reparación de los equipos; para realizar los cálculos citados se requiere la integridad de estos datos: primero deben cumplir con las definiciones de la confiabilidad; para su manejo debe estar previsto un procedimiento de almacenamiento idóneo; además se debe analizar el tamaño de la población de la muestra, la cual debe ser representativa para un análisis estadístico (BS-EN, 2016); por lo tanto es de suma importancia el diseño de formatos detallados para su recolección.

Otro punto importante antes de comenzar a calcular los indicadores de la confiabilidad es realizar una delimitación y estructuración jerárquica de los sistemas o equipos. En esta delimitación se debe establecer donde empiezan y terminan los sistemas industriales, la norma ISO 14224 (recolección e intercambio de datos de confiabilidad y mantenimiento de equipos) nos muestra ejemplos claros sobre este punto (ISO, 2016). Un grupo electrógeno puede tomarse como un sistema, o se lo puede dividir, un ejemplo se muestra en la figura $\mathrm{N} 2$.

Figura 2. Ejemplo de límites en aplicado a grupos electrógenos (BS-EN, 2016).
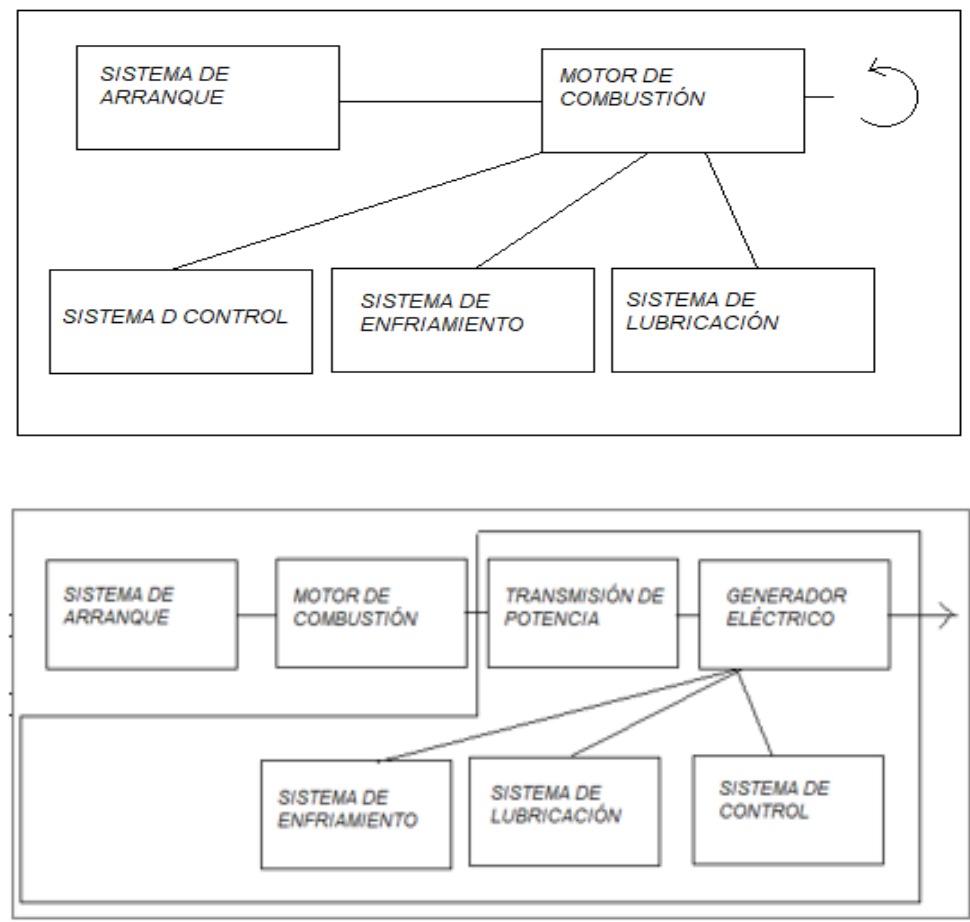

Fuente: ISO 14224 
Es importante que los equipos tengan un código único que nos ayude a identificarlos y nos permita hacer registros de los eventos como fallos e intervenciones de mantenimiento (GARCIA, 2017), para ello el primer paso es desarrollar una estructura jerárquica de los niveles del inventario técnico. La norma ISO-14224 presenta una estructura para la taxonomía de equipos (ISO, 2016). Par fines prácticos la jerarquía de equipos se los resume en la Figura 3.

Figura 3. Niveles jerárquicos Norma 14224.
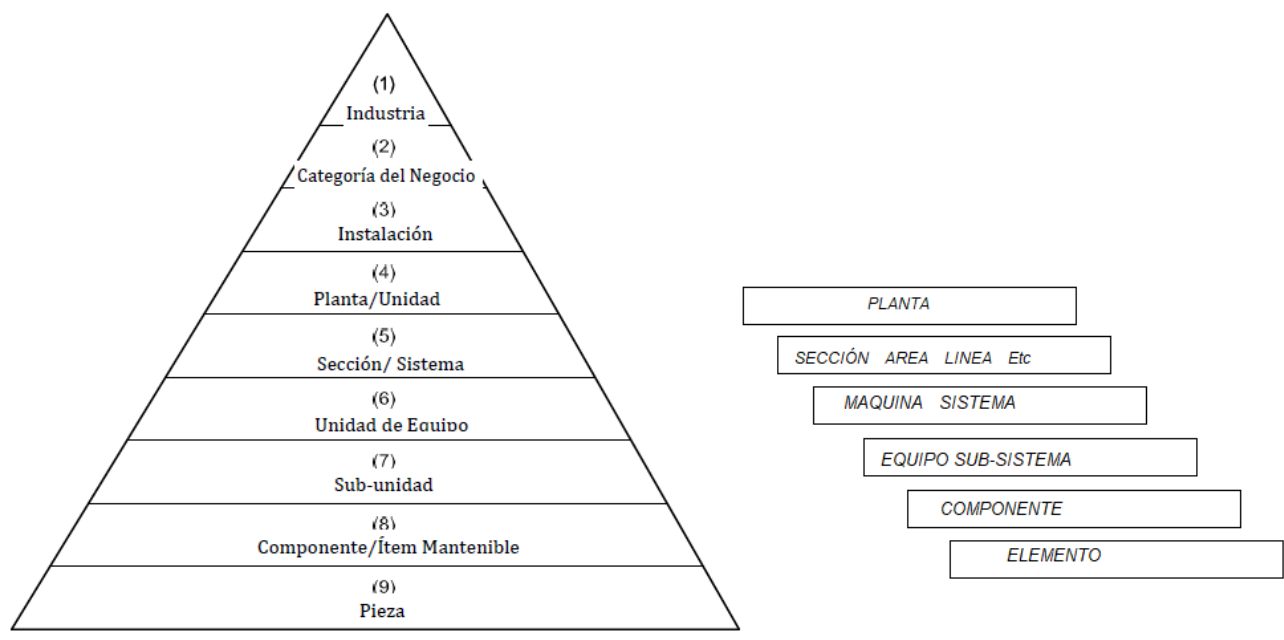

Fuente: Norma ISO 14224

El sistema de codificación consiste en asignar un numero de dígitos en cada nivel jerárquico, estos pueden ser una combinación de letras y números y el resultado es un código único (GARCIA, 2017), Figura 4.

Figura 4. Ejemplo de un sistema de codificación

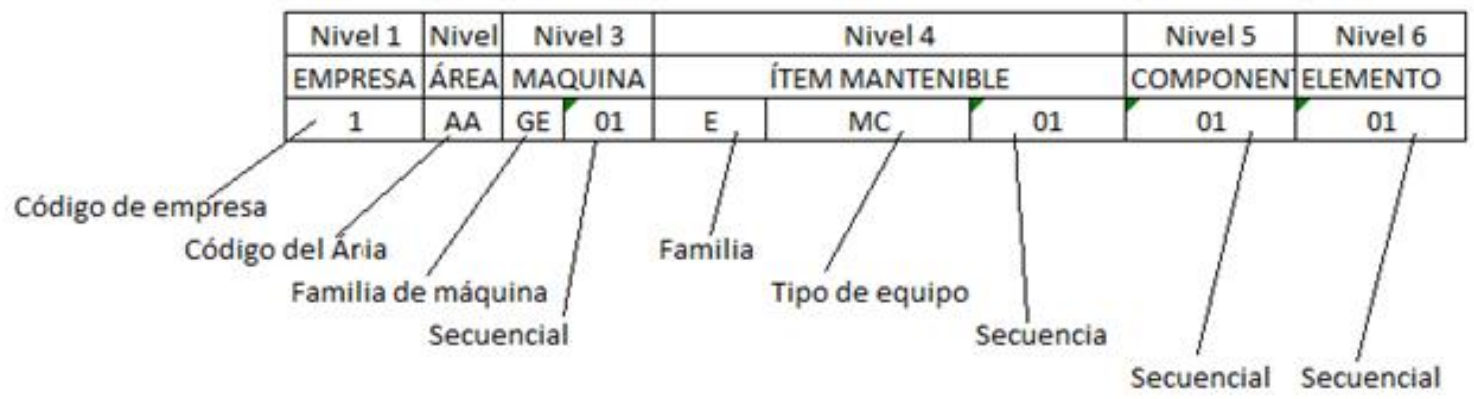

Fuente: Elaboración propia.

Para recolectar los datos necesarios utilizados en los cálculos de los indicadores de fiabilidad, mantenibilidad y disponibilidad, se deben diseñar tablas con todos los campos 
requeridos, la norma ISO 14224 presenta una ficha para el registro de la información base, ver Tabla 1.

Tabla 1. Recolección de datos con fines del cálculo de indicadores RAMS.

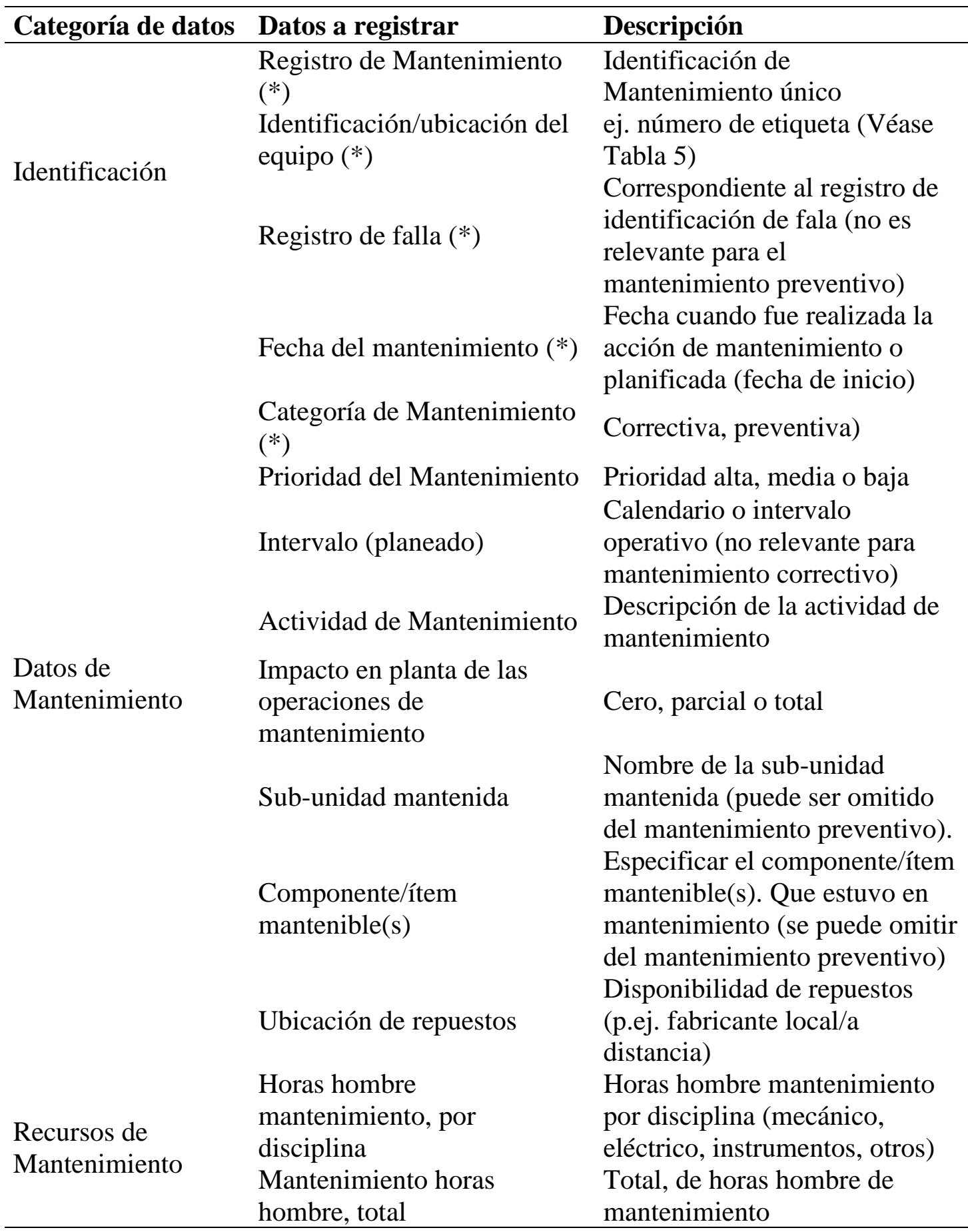

Fuente: ISO 14224

Para el estudio del análisis RAM se deben conocer las funciones estadísticas relacionadas con los estudios del fallo y la optimización de los tiempos de reparación, entre ellas 
tenemos: la función de la densidad de probabilidad $\mathrm{f}(\mathrm{t})$, la función de distribución acumulada de la probabilidad $\mathrm{F}(\mathrm{t})$, función de la fiabilidad $\mathrm{R}(\mathrm{t})$ y función de la tasa de fallos $\lambda(\mathrm{t})$ (CRESPO, MOREU, \& SANCHEZ, 2004).

Las expresiones de las distribuciones acumuladas más utilizadas para el cálculo de la fiabilidad y mantenibilidad se describen en la Tabla 3 (NACHIAS, 1995) (ACUÑA, 2003) (KNEZEVIC, 1966) (MERUANE, 2014)

Tabla 2. Distribuciones acumuladas de probabilidad.

\begin{tabular}{ccc}
\hline Distribución & Función & Dominio \\
\hline Exponencial & $1-\exp (\mathrm{t} / \square)$ & $\mathrm{t} \geq 0$ \\
Normal & $\phi[(\mathrm{t}-\square) / \beta]$ & $-\infty<\mathrm{t}<+\infty$ \\
Log.Normal & $\phi[\ln (\mathrm{t}-\mathrm{\gamma})-\alpha) / \beta]$ & $\mathrm{t} \geq \mathrm{v}, \mathrm{t} \geq 0$ \\
Weibull & $1-\exp -[(\mathrm{t}-\mathrm{s}) /(\alpha-\mathrm{s})] \beta$ & $\mathrm{t} \geq \mathrm{y}, \mathrm{t} \geq 0$ \\
\hline
\end{tabular}

Fuente: J. NACHIAS, Fiabilidad, Madrid: Isdefe

Una comparación del comportamiento de las funciones estadísticas en el tiempo se representa en la Figura 5. (Red Temática Nacional Sobre Seguridad de Funcionam, 2010)

Figura 5. Comparación de las funciones estadísticas

Modelo

Fuente: Red Temática Nacional Sobre Seguridad de Funcionamiento, Aplicaciones a la Confiabilidad, 
Por su adaptabilidad la distribución de Weibull es la más utilizada para cálculos de fiabilidad y mantenibilidad, pues posee parámetros que se adaptan a los diferentes estados de la vida útil de los equipos (Red Temática Nacional Sobre Seguridad de Funcionamiento 2010) (PARRA \& CRESPO , 2016); muy utilizada en el estudio de tiempos de vida útil o tiempos para la falla en elementos mecánicos; una característica muy importante de la distribución del Weibull es que puede tomar propiedades de otras distribuciones dependiendo de los valores que toman sus parámetros. (YANEZ, GOMEZ, \& VALBUENA, 2004).

Las fórmulas de distribución acumulada para los cálculos de la Fiabilidad (R), la Infiabilidad (F) y La Mantenibilidad (M) en función de los parámetros, $\alpha$ (parámetro de escala) y $\beta$ (parámetro de forma) son:

$$
\mathrm{F}(\mathrm{t})=1-\mathrm{e}^{\wedge}\left(-[\mathrm{t} / \alpha]^{\wedge} \beta\right) ; \mathrm{R}(\mathrm{t})=\mathrm{e}^{\wedge}\left(-[\mathrm{t} / \alpha]^{\wedge} \beta\right)=\mathrm{M}(\mathrm{t})=1-\mathrm{e}^{\wedge}\left(-[\mathrm{t} / \alpha]^{\wedge} \beta\right)
$$

\section{Metodología.}

Una metodología para el desarrollo de los análisis RAM se resume en los siguientes pasos (MARTINEZ, 2018):

Registro de datos: tiempo entre fallos, tiempos de Operación, tiempo fuera de servicio por fallos) (CRESPO, MOREU, \& SANCHEZ, 2004) (MERUANE, 2014) (HARRIS, 2000). Para el registro de los tiempos operativos entre fallos es recomendable que los equipos cuenten con horómetros, estos datos deben tomarse diariamente. Para el registro de los tiempos de reparación es necesario anotar las fechas y horas de inicio y fin de la reparación, las horas hombre dedicadas a mantenimiento por diciplina, la demora de los tiempos logísticos y administrativos, no se debe tomar en cuenta los tiempos que no estén relacionados con el mantenimiento, en el registro debe anotarse las tareas realizadas y qué componente fue afectado.

Comprobación de la valía de los datos (eliminación de factores extremos). Hay que tener cuidado con los datos atípicos, generalmente muy alejados de la media y su desviación estándar, ejemplo los tiempos utilizados para reparaciones mayores (overhaul).

Comprobar el ajuste de la distribución. Prueba realizada para determinar a qué tipo de distribución se ajustan los datos, para lo cual es necesario realizar la linealización y se debe verificar la distribución que tiene el mejor índice de determinación.

Cálculo de los índices de fiabilidad y mantenibilidad utilizando la distribución más adecuada.

Determinación del parámetro $\beta$ (parámetro de forma) de la distribución de Weibull para intuir la etapa en que se encuentra la vida del equipo (curva de la bañera) 
Cálculo de la disponibilidad en función del tiempo medio entre fallos y del tiempo medio para reparar.

\section{Resultados.}

Luego de la recolección de los tiempos entre fallos y los tiempos de reparación de los 51 grupos electrógenos prime seleccionados para el estudio por un período de once meses, a los datos se les realizó la prueba de puntuación $\mathrm{Z}$ para determinar si existen valores atípicos, que estén muy alejados de la media y su desviación estándar, a ecuación 2

$$
\mathrm{Z}=(\text { Dato evaluado }-\mathrm{m}) / \sigma(2)
$$

Donde:

$\mathrm{m}=$ media

б= Desviación Estándar

La puntuación $Z$ es atípica cuando su valor es menor a -3 o mayor a 3.

Si un valor es considerado atípico es muy importante verificar los registros realizados en la recolección de datos para encontrar errores, si no existen errores los datos pueden ser validados nuevamente para los cálculos de la confiabilidad.

Los datos válidos para continuar con él análisis se muestran en la Tabla 3

Tabla 3. Datos tiempos de operación y reparación.

\begin{tabular}{|c|c|c|c|c|c|c|c|c|c|c|c|c|c|c|}
\hline \multicolumn{15}{|c|}{ Tiempos entre fallos horas } \\
\hline 2645 & 2512 & 3169 & 3725 & 3078 & 1783 & 3842 & 3943 & 3896 & 252 & 3026 & 1658 & 855 & 821 & 2282 \\
\hline 851 & 330 & 4037 & 3495 & 2772 & 3326 & 3173 & 1220 & 1819 & 76 & 3800 & 4467 & 2025 & 723 & 3915 \\
\hline 4315 & 1777 & 3596 & 1206 & 3442 & 990 & 183 & 2153 & 4114 & 919 & 838 & 4181 & 2648 & 1523 & 4463 \\
\hline 3267 & 588 & 3340 & 704 & 4070 & 706 & 2441 & 1187 & 1468 & 2705 & 2163 & 2866 & 3892 & 3779 & 757 \\
\hline 1624 & 3755 & 800 & 2996 & 2461 & 2554 & 1804 & 4137 & 3304 & 1370 & 691 & 1135 & 3372 & 1334 & 3982 \\
\hline 614 & 713 & 4008 & 3452 & 3402 & 190 & 3198 & & & & & & & & \\
\hline \multicolumn{15}{|c|}{ Tiempo de reparación en horas } \\
\hline 0.27 & 0.32 & 0.32 & 0.50 & 0.50 & 0.50 & 0.50 & 0.50 & 0.75 & 1.00 & 1.00 & 1.00 & 1.00 & 1.00 & 1.00 \\
\hline 1.00 & 1.42 & 1.50 & 1.67 & 2.00 & 2.02 & 2.50 & 2.75 & 3.00 & 3.00 & 3.00 & 3.32 & 4.00 & 4.50 & 5.00 \\
\hline 6.00 & 6.00 & 6.32 & 7.00 & 7.40 & 7.60 & 7.87 & 8.00 & 8.12 & 8.20 & 8.25 & 8.30 & 8.48 & 8.50 & 8.82 \\
\hline & & & & & 10.3 & 10.5 & 10.6 & 11.2 & 13.0 & 14.0 & 14.7 & 14.9 & 15.4 & 15.7 \\
\hline 9.00 & 9.00 & 9.15 & 9.62 & 9.92 & 0 & 2 & 2 & 2 & 0 & 5 & 7 & 7 & 2 & 5 \\
\hline 15.9 & 16.3 & 16.4 & 16.5 & 16.8 & 17.2 & 17.3 & 19.5 & 19.9 & 21.0 & 21.3 & 21.4 & 21.4 & 22.2 & 22.2 \\
\hline 2 & 2 & 5 & 7 & 2 & 2 & 0 & 2 & 0 & 2 & 7 & 2 & 7 & 0 & 5 \\
\hline 22.4 & 22.5 & 22.6 & 22.7 & 23.2 & 24.0 & 47.5 & & & & & & & & \\
\hline 2 & 0 & 2 & 0 & 2 & 0 & 0 & & & & & & & & \\
\hline
\end{tabular}

Fuente: Elaboración propia. 
El siguiente paso fue realizar las linealizaciones para las distribuciones, exponencial y Weibull, que son las más utilizadas en el contexto de la confiabilidad, con esto se verificó a qué tipo de distribución se ajustan los datos. El criterio utilizado para esta evaluación es el índice de determinación el cual indica que porcentaje de los puntos (coordenadas) están relacionados linealmente, identificado como R2.

\section{Linealización Distribución De Weibull.}

La Ecuación bi-paramétrica para el cálculo de la fiabilidad es:

$$
\mathrm{R}(\mathrm{t})=\mathrm{e}-(\mathrm{t} / \alpha) \beta
$$

Aplicando los logaritmos neperianos a la función $R(t)$ por dos ocasiones obtenemos:

$$
\ln \ln \{1 /[1-\mathrm{R}(\mathrm{t})]\}=\beta \ln (\mathrm{t})-\beta \ln \alpha
$$

La expresión anterior se la compara con la ecuación de una recta.

$$
\mathrm{Y}=\mathrm{Bx}+\mathrm{C}
$$

Donde:

$$
\begin{array}{r}
\mathrm{Y}=\ln \ln \{1 /[1-\mathrm{R}(\mathrm{t})]\} \\
\mathrm{Bx}=\beta \ln (\mathrm{t}) \\
\mathrm{C}=-\beta \ln \alpha \quad(8)
\end{array}
$$

Para obtener las coordenadas para la linealización, se aplica:

Para el eje (Y): $\quad \ln [\ln (1 / 1-R a n g o$ Mediana) $]$

Para el eje $(\mathrm{X}): \quad \ln (\mathrm{t}) \quad(10)$

Una manera de realizar aproximaciones es por medio del rango de las medianas, que ayuda al cálculo de las probabilidades acumuladas, para ello utilizamos los estimadores matemáticos como las fórmulas de Bernard, estas fórmulas dependen del tamaño de la muestra a analizar:

$\mathrm{N}>50$

$$
\mathrm{M}=\mathrm{i} / \mathrm{M}
$$

$50>\mathrm{N}>20$

$$
\mathrm{M}=\mathrm{i} / \mathrm{N}+1
$$

$\mathrm{N}<20$

$$
\mathrm{M}=\mathrm{i}-0.3 / \mathrm{N}+0.4
$$

Donde:

$\mathrm{i}=$ Numero de orden de la muestra 
$\mathrm{N}=$ Tamaño de la muestra

$\mathrm{M}=$ Rango de las medianas

Los resultados de la linealización para la distribución de Weibull son:

Recta de linealización $\mathrm{Y}=1.4176 \mathrm{X}-11.17$

Parámetro de forma $B=\beta=1.4117$

Intercepto

$\mathrm{C}=-11.17$

Índice de determinación

$\mathrm{R} 2=0.93$

\section{Linealización Distribución De Exponencial}

La función de la fiabilidad para la distribución exponencial es:

$R(t)=e(-\lambda t)$

Donde:

$\lambda=$ Tasa de fallos $=1 /$ MTBT

$\mathrm{t}=$ Tiempo hasta el fallo

Las coordenadas para la linealización exponencial son:

$\mathrm{Y}=\operatorname{Ln}[1-\mathrm{F}(\mathrm{t})]$

$\mathrm{X}=$ tiempo operativo hasta el fallo

Donde:

$\mathrm{F}(\mathrm{t})=$ Función de densidad de probabilidad

$\mathrm{Ln}=$ Logaritmo natural

$\mathrm{MTBF}=$ Tiempo medio entre fallos

Los resultados para la linealización de la distribución exponencial son:

Tasa de fallos

$\lambda=0.000532926$

Tiempo medio entre fallos

$\mathrm{MTBF}=1876.431$

Recta de linealización

$Y=-0.000532926 X+0.3493867$ 
Índice de determinación

$\mathrm{R} 2=0.79891$

Con estos datos se obtuvieron las rectas de linealización para las distribuciones analizadas:

Figura 6. Comparación Índices de Determinación
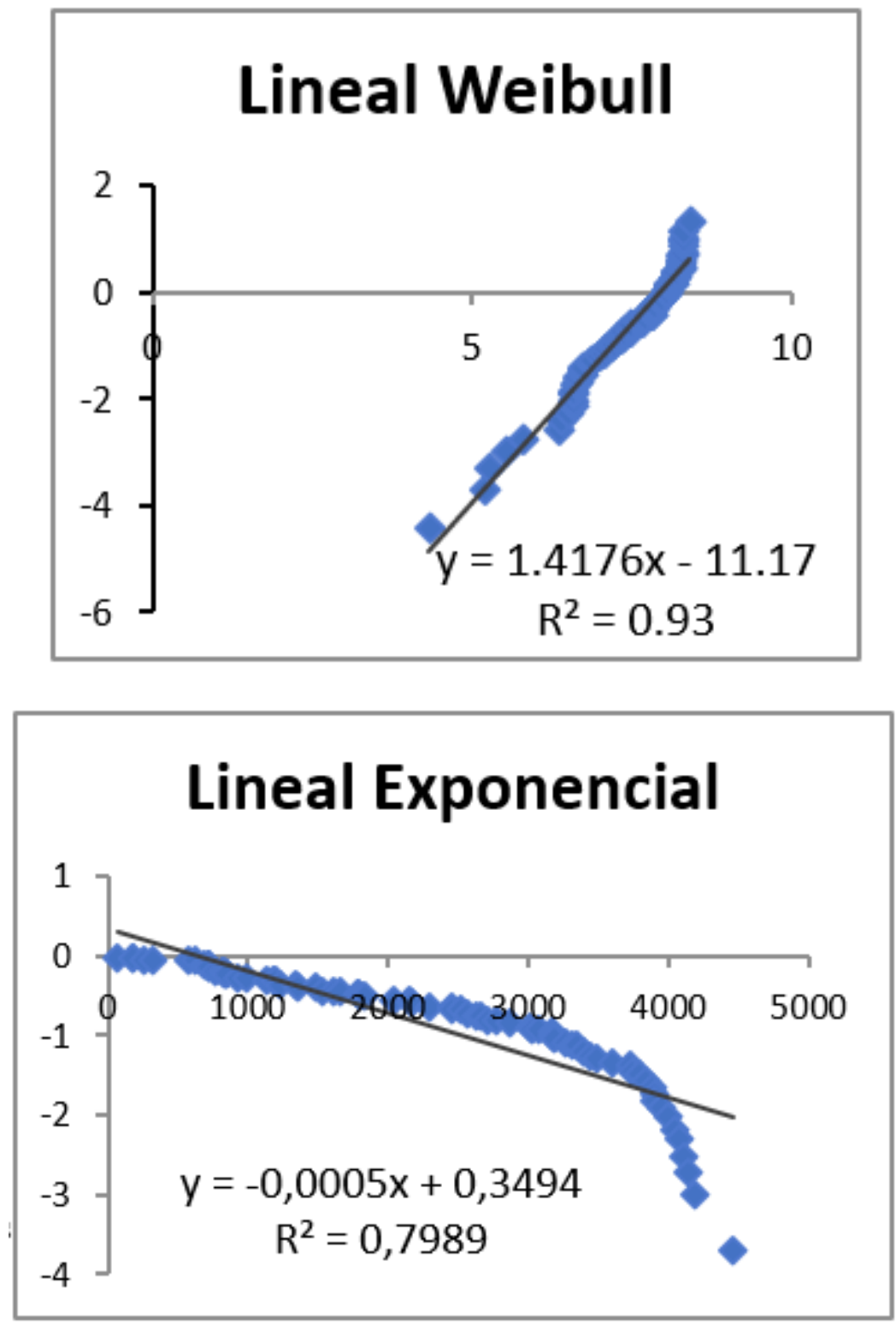

Fuente: Elaboración propia.

Para seleccionar el modelo de distribución más adecuado se calcularon los coeficientes de determinación. La distribución seleccionada es la de Weibull, su índice de determinación es superior, R2=0.93.

Con la ecuación de la recta de linealización de la distribución de Weibull Figura 6, se tiene la pendiente de la recta, que es el parámetro de forma $\beta$ de la función de distribución, 
y con el punto de intersección de la recta con el eje y (el punto C), podemos obtener el parámetro de escala $\alpha$ con la expresión:

$\alpha=\mathrm{e}(-\mathrm{C} / \beta) \quad(15)$

$\alpha=2845,77684$

Con los parámetros $\alpha$ y $\beta$ de la distribución de Weibull se calcula la función de la Fiabilidad $R(t)$ reemplazando varios valores del tiempo (t) Figura 7.

Las ecuaciones para el cálculo de la Fiabilidad e Infiabilidad para la distribución de Weibull son:

Fiabilidad

$$
\mathrm{R}(\mathrm{t})=\mathrm{e}-[\mathrm{t} / \alpha] \beta
$$

Infiabilidad

$$
F(t)=1-e-[t / \alpha] \beta
$$

Figura 7. Curva de la fiabilidad.

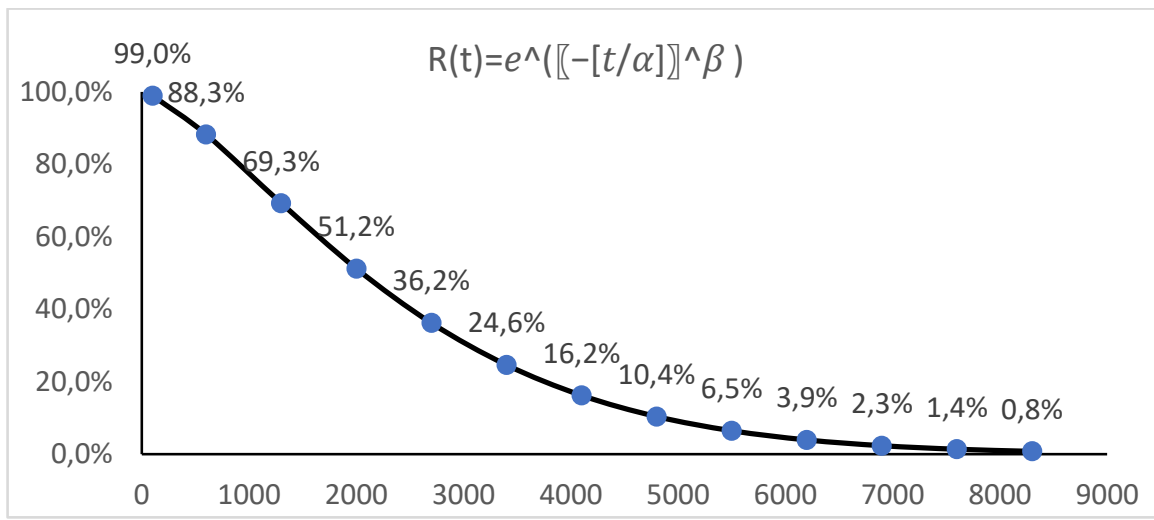

Fuente: Elaboración propia.

Para calcular el Tiempo medio entre fallas MTBF con los parámetros de la distribución de Weibull de aplica la distribución Gamma.

$$
\mathrm{MTBF}=\alpha \times \Gamma(1+1 / \beta)(18)
$$

$\mathrm{MTBF}=2405$

Se repite el mismo procedimiento para el cálculo de la mantenibilidad, se debe utilizar los datos de los tiempos de reparación. La expresión utilizada para Mantenibilidad es: 
$\mathrm{F}(\mathrm{t})=1-\mathrm{e}-[\mathrm{t} / \alpha] \beta$

El tiempo medio para reparar en función de los parámetros de Weibull se obtienen con la expresión:

$\operatorname{MTTR}=\alpha \times \Gamma(1+1 / \beta)(20)$

$\operatorname{MTTR}=9.99$

La Disponibilidad se calcula en función del MTBF Tiempo Medio entre Fallos y el MTTR Tiempo Medio para Reparar.

La ecuación para la disponibilidad intrínseca (por diseño) es:

Disponibilidad $=\mathrm{MTBF} /(\mathrm{MTBF}+\mathrm{MTTR})$

Disponibilidad $=2405 /(2405+10) \times 100=99.5 \%$

Un resumen de los cálculos probabilísticos de la fiabilidad, infiabilidad se presentan en las Figura 8.

Figura 8. Curva de la Fiabilidad e Infiabilidad

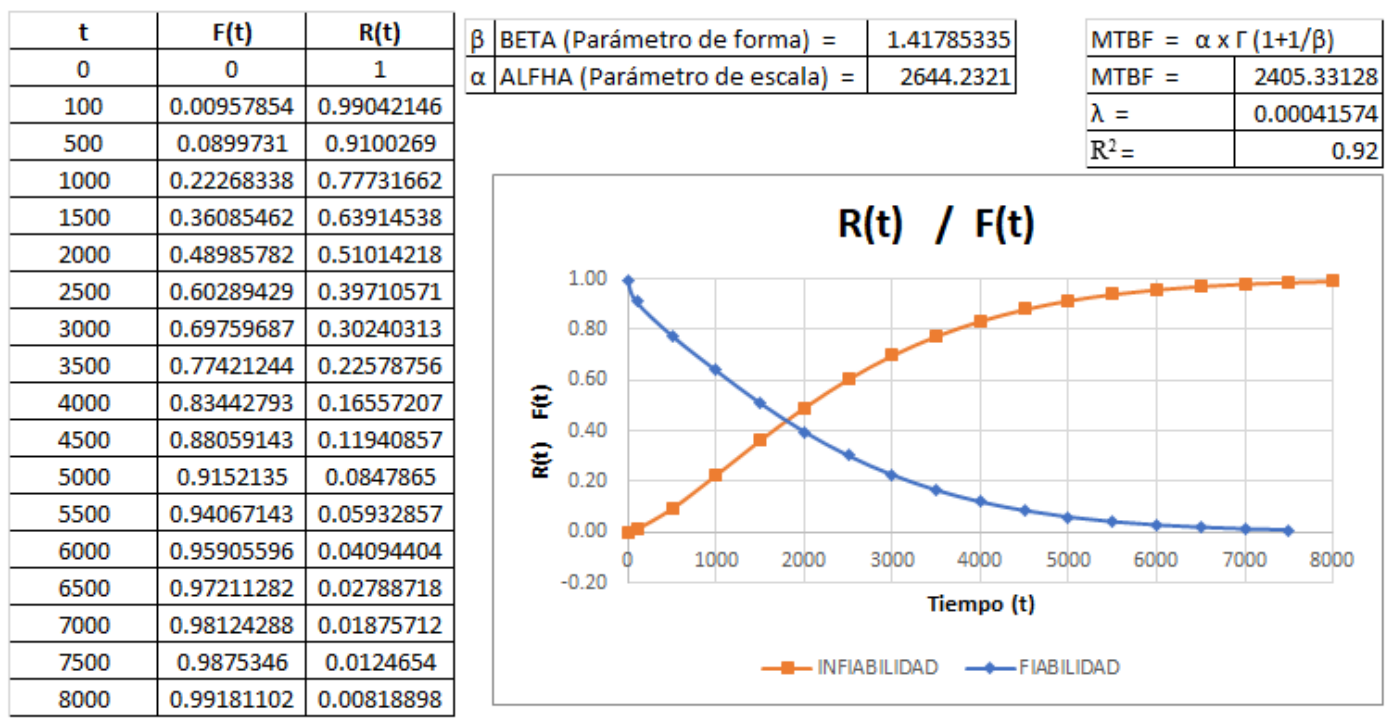

Fuente: Elaboración propia.

Los cálculos de la mantenibilidad en forma son iguales a los de la Infiabilidad, con la diferencia que se trabajan con los tiempos de reparación.

Un resumen de los cálculos de la mantenibilidad se presenta en la figura 9. 
Figura 9. Curva de la Mantenibilidad.

\begin{tabular}{c|c}
$\mathbf{X}$ & $\mathbf{Y}$ \\
\hline $\mathbf{t}$ & $\mathbf{M}(\mathbf{t})$ \\
\hline 0 & 0 \\
\hline 2 & 0.20741329 \\
\hline 4 & 0.35825733 \\
\hline 6 & 0.47654994 \\
\hline 8 & 0.57105087 \\
\hline 10 & 0.64730016 \\
\hline 12 & 0.70922483 \\
\hline 14 & 0.75975417 \\
\hline 16 & 0.80113602 \\
\hline 18 & 0.83512644 \\
\hline 20 & 0.86311433 \\
\hline 22 & 0.88620799 \\
\hline 24 & 0.90529799 \\
\hline 26 & 0.92110374 \\
\hline 28 & 0.934209 \\
\hline 30 & 0.94508918 \\
\hline 32 & 0.95413265 \\
\hline 34 & 0.96165753 \\
\hline &
\end{tabular}

\section{$\beta \quad$ BETA (Parámetro de forma) $=\mid 0.93221361$ $\alpha$ ALFHA (Parámetro de escala) $=9.56690316$ \\ MTTR $=\alpha \times \Gamma(1+1 / \beta)$

\begin{tabular}{l|r|}
\hline $\mathrm{MTTR}=$ & 9.8821713 \\
\hline $\mathrm{R}^{2}=$ & 0.93 \\
\hline
\end{tabular}

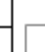

MANTENIBILIDAD

Fuente: Elaboración propia.

\section{Discusión.}

El resultado obtenido al calcular el índice de determinación de la distribución de Weibull es mayor al índice de determinación obtenido para la distribución exponencial lo que garantiza un mejor ajuste de los datos para la distribución de Weibull para el cálculo de los indicadores RAM.

Con los parámetros de la distribución de Weibull se calcula la probabilidad que el equipo funcione correctamente para cualquier tiempo de ensayo, ejemplo a las 1,000 horas la probabilidad que un equipo funcione correctamente es del $77.77 \%$, y la probabilidad de fallo será por lo tanto de $22.23 \%$, ver Figura 8. Con esto es posible estimar la probabilidad que un equipo falle en un período de tiempo, el cual será seleccionado de acuerdo a las necesidades del estudio a realizar.

Para el cálculo de la mantenibilidad se operó con la misma metodología, ejemplo la probabilidad que un equipo esté reparado a las 10 horas es del 64\%, a las 20 horas es $86 \%$ ver Figura 9. En el caso del estudio de la mantenibilidad se puede pronosticar la probabilidad de que un equipo esté reparado en un cualquier lapso de tiempo de interés, indicador muy válido para evaluar estrategias que permitan disminuir los tiempos de reparación.

El parámetro de forma $\beta$ de la distribución de Weibull para el cálculo de la fiabilidad, índica en que parte de la vida se encuentran los elementos (curva de la bañera), en el estudio realizado el valor de $\beta$ es 1.39 lo que indica que los equipos no han entrado la etapa de envejecimiento que se caracteriza por el aumento de la tasa de fallos. En la 
práctica el parámetro $\beta$ debe superar el valor de 3 para considerar que los equipos se encuentran en la etapa de envejecimiento.

El tiempo medio entre fallos obtenido es 2405 horas (cada 2405 horas se espera que uno de los 51 equipos analizados falle) y el tiempo medio para la reparación es de 10 horas. Los tiempos de operación son muy superiores a los tiempos de reparación para el estudio realizado, la disponibilidad total calculada es del 99,5\%. Esta información confirma que la mayoría los equipos no han entrado en la zona de desgaste (el tiempo medio entre fallas es alto y los tiempos de reparación son bajos).

\section{Conclusiones:}

- El paso primordial para el cálculo de los indicadores es la validez de los datos, es necesario implementar un método con formatos definidos para la recolección de la información de los indicadores RAM (tiempo operativo entre fallos y los tiempos necesarios para la reparación).

- El tiempo de reparación de un equipo está dividido en: tiempo para la detección de la falla, tiempo logístico para conseguir y organizar los recursos necesarios, tiempo activo en la reparación, tiempos de pruebas e incluso tiempos de traslado. Para el análisis de la mantenibilidad es necesario incluirlos todos.

- Los estudios de la fiabilidad pueden enfocarse tomando varios criterios, por ejemplo, se pueden calcular para flotas o grupos de equipos o para cada equipo, también se los puede analizar para un determinado modo de fallo o para todos los modos de falla ocurridos, esta variedad de posibilidades proporcionará información diferente, lo cual es un apoyo importante para la toma de estrategias para mejorar la fiabilidad de los equipos.

- Los indicadores de fiabilidad y mantenibilidad proporcionan información general válida, determinan hacia donde debemos dirigir los esfuerzos para la conservación adecuada de las funciones de los equipos, dichas acciones deben estar encaminadas alargar los tiempos entre fallos y disminuir los tiempos de reparación, para afirmar estas estrategias se deben apoyar en otras metodologías proactivas como el Análisis Causa Raíz, Análisis de los Modos de Fallos y Efectos, Mantenimiento Centrado en Fiabilidad, Análisis de Pareto, etc.

\section{Referencias bibliográficas:}

Acuña, J. (2003). Ingeniería de la Confiabilidad. Costa Rica: Tecnológica de Costa Rica. BS-EN. (2016). ISO 14224 Recolección e intercambio de datos de confiabilidad y mantenimiento de equipos. BSI Standards Limited.

CEN. (Julio de 2018). Terminología de mantenimiento. EN-13306. ARENOR. 
Crespo, A., Moreu, P., \& Sánchez, A. (2004). Ingeniería de Mantenimiento. Madrid: AENOR.

Deparment Of Defense, U. (3 de Agosto de 2005). Guía para lograr la Fiabilidad, Disponibilidad y Mantenibilidad. USA: Deparment of Defense .

Depatment Of Defense, U. (24 de Mayo de 1966). MILI-HDBK-472. Manintainability Predicction. Washington, USA: DEPATMENT OF DEFENSE, USA.

GARCIA, O. (2017). Gestión Moderna del Mantenimiento Industrial; Principios fundamentales. Ediciones de la U.

Harris, M. (2000). Gestión del Mantenimiento Industrial. Madrid: Fundación REPSOL.

Knezevic, J. (1966). Mantenimiento. (4ta, Ed.) Madrid: Isdefe.

Martinez, E. M. (2018). Seminario de Confiabilidad.

Meruane, V. (2014). Gestión de Activos. Santiago: DEMEC.

Moubray, J. (2002). Mantenimiento Centrado en la confiabilidad. Asheville: Industrias press Inc.

Nachias, J. (1995). Fiabilidad. Madrid: Isdefe.

Parra, C., \& Crespo , A. (2016). Métodos de análisis de fiabilidad, mantenibilidad, disponibilidad y riego. Ingecom.

Red Temática Nacional Sobre Seguridad de Funcionam. (2010). Aplicaciones a la Confiabilidad, Aplicaciones Prácticas. Madrid: Ingeman.

Yanez, M., Gomez, H., \& Valbuena, G. (2004). Ingeniería de la confiabilidad y análisis probabilístico del riesgo. Reliability and risk managemeny S.A. 


\section{PARA CITAR EL ARTÍCULO INDEXADO.}

Gallegos Londoño, C. M., Viscaíno Cuzco, M. A., \& Sergio Raúl, S. R. (2020). Estudio de fiabilidad, mantenibilidad y disponibilidad aplicado a grupos electrógenos prime . ConcienciaDigital, 3(3), 44-61. https://doi.org/10.33262/concienciadigital.v3i3.1266

\section{Liencia}

El artículo que se publica es de exclusiva responsabilidad de los autores y no necesariamente reflejan el pensamiento de la Revista Ciencia Digital.

El artículo queda en propiedad de la revista y, por tanto, su publicación parcial y/o total en otro medio tiene que ser autorizado por el director de la Revista Ciencia Digital.

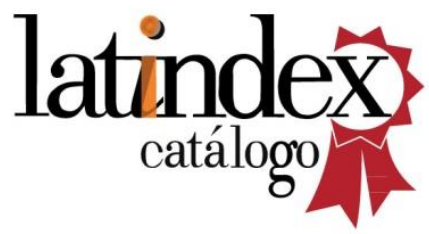

